\title{
NON-GRAY PHONON TRANSPORT USING A HYBRID BTE-FOURIER SOLVER
}

\author{
James M. Loy, Dhruv Singh and Jayathi Y. Murthy \\ School of Mechanical Engineering, Purdue University \\ West Lafayette, Indiana 47907-2088
}

\begin{abstract}
Non-gray phonon transport solvers based on the Boltzmann transport equation (BTE) are frequently employed to simulate sub-micron thermal transport. Typical solution procedures using sequential solution schemes encounter numerical difficulties because of the large spread in scattering rates. For frequency bands with very low Knudsen numbers, strong coupling between the directional BTEs results in slow convergence for sequential solution procedures. In this paper, we present a hybrid BTE-Fourier model which addresses this issue. By establishing a phonon group cutoff (say $\mathrm{Kn}=0.1$ ), phonon bands with low Knudsen numbers are solved using a modified Fourier equation which includes a scattering term as well as corrections to account for boundary temperature slip. Phonon bands with high Knudsen numbers are solved using a BTE solver. Once the governing equations are solved for each phonon group, their energies are then summed to find the total lattice energy and correspondingly, the lattice temperature. An iterative procedure combining the lattice temperature determination and the solutions to the modified Fourier and BTE equations is developed. The procedure is shown to work well across a range of Knudsen numbers.
\end{abstract}

\section{NOMENCLATURE}

$C_{\omega, p} \quad$ phonon volumetric specific heat $\left[\frac{J}{m^{3} K}\right]$

$e^{\prime \prime}{ }_{\omega, p} \quad$ phonon energy density $\left[\frac{J}{m^{3} s r}\right]$

$e_{\omega, p}^{0} \quad$ phonon band contribution to total lattice energy $\left[\frac{J}{m^{3} s r}\right]$

$\bar{e}_{\omega, p} \quad$ angular average of band energy density $\left[\frac{J}{m^{3} s r}\right]$

$f^{0} \quad$ equilibrium phonon distribution

$\hbar \quad$ reduced Planck's constant $[J S]$

$k_{\omega, p} \quad$ thermal conductivity of phonon band $\left[\frac{W}{m K}\right]$

Kn $\quad$ Knudsen number $\left(=\frac{v_{\omega, p} \tau_{\omega, p}}{L}\right)$
$\vec{K} \quad$ wave vector $\left[\mathrm{m}^{-1}\right]$

$L \quad$ characteristic length $[m]$

$\vec{q}_{\omega, p}^{\prime \prime} q_{\omega, p}$ heat flux vector associated with phonon band $\left[\frac{W}{m^{2}}\right]$

$\vec{r} \quad$ position vector $[\mathrm{m}]$

$\hat{\mathrm{s}}$ direction vector

$T_{L} \quad$ lattice temperature $[K]$

$T_{\omega, p} \quad$ phonon band temperature $[K]$

$T_{\text {ref }} \quad$ reference temperature $[K]$

$\vec{v}_{\omega, p} \quad$ phonon group velocity $[\mathrm{m} / \mathrm{s}]$

Greek

$\omega \quad$ angular frequency $[\mathrm{rad} / \mathrm{s}]$

$\Delta \Omega \quad$ discrete solid angle $[s r]$

$\tau_{\omega, p} \quad$ phonon scattering rate $[s]$

$\Phi_{\omega, \mathrm{p}} \quad$ perturbation $\left[\frac{J}{m^{3} s r}\right]$

\section{Subscripts}

$\begin{array}{ll}\omega & \text { advancing contact angle } \\ L & \text { lattice } \\ p & \text { polarization } \\ \text { wall } & \text { associated with wall }\end{array}$

\section{INTRODUCTION}

In the past few decades, the extreme miniaturization of integrated circuits and the advent of nanotechnology has increased interest in understanding thermal transport at submicron scales [1]. Thermal transport in semiconductors and dielectrics is almost exclusively due to phonons, which are quanta of lattice vibrations [2]. Many different computational approaches have been taken to analyze sub-micron thermal transport phenomena including molecular dynamics (MD) [35], atomistic Green's function (AGF) approaches to capture wave effects and transmission across interfaces [6,7], and 
analyses based on the phonon Boltzmann transport equation (BTE) to capture bulk, impurity, and boundary scattering [2,810,12-15].

When domain length scales become competitive with the carrier mean free path and phase coherence effects can be neglected, particle-based approaches based on the phonon Boltzmann transport equation may be used. BTE simulations have been used for carrier simulation in a variety of applications, including electron and phonon transport in semiconductor devices, and in the simulation of rarefied gas dynamics [15, 8-10]. The radiative transfer equation (RTE) for participating media [11] has a similar structure, and solution methods developed for radiation may be used to solve the BTE as well.

Several variants of the phonon BTE with differing degrees of complexity have been developed to calculate thermal transport in nanocomposites [9], thin films [8], and microelectronics $[10,11]$. The simplest case is the gray model [12]. In this model, all phonons are grouped into a single "gray phonon," without accounting for the effects of phonon dispersion and polarization. This model can capture the broad behavior of phonon transport, including ballistic and boundary scattering effects, but cannot capture the granularity of phonon behavior, which is critical in microelectronics applications where electron-phonon scattering channels energy into specific phonon groups [11]. The next level of complexity is the socalled "two-fluid" model, or the semi-gray model [13, 14]. Here, the phonons are grouped into one of two groups: a propagating group and a reservoir group. The reservoir group is responsible only for modeling thermal capacitance, and is assigned a zero group velocity; the propagating group is responsible for thermal transport. The success of this type of model depends critically on the relaxation time specified for scattering between the two groups. Depending on the group velocity assigned to the propagating mode, the relaxation time must be adjusted to match bulk properties; improper choices lead to long relaxation times and untenably high temperature predictions in transistor simulations [8]. More recently, phonon transport models have been proposed which account for the granularity of phonon transport $[8,11,17]$ by incorporating phonon frequency and polarization dependence [12, 8]. Wang [17] developed three-phonon scattering rate expressions fully accounting for energy and momentum conservation rules. $\mathrm{Ni}$ [11] developed a single-mode relaxation approximation to these scattering rate expressions, retaining dispersion and polarization dependence. He coupled the phonon BTE to a Monte Carlo electron transport simulation of a bulk transistor, and predicted the thermal field in a transistor. These models are all guaranteed to default to the Fourier conduction model in the limit of low Knudsen number (inverse acoustic thickness).

Though these detailed non-gray phonon transport models can capture the details of thermal transport well, they are difficult to solve because of the large spread in relaxation times. In a typical transport problem in room-temperature silicon, single-mode relaxation times may range of several orders of magnitude, rendering some phonon groups nearly- ballistic, which other may be acoustically very thick. Typical discrete ordinates or finite volume solution methods, borrowed from the radiation literature [10], employ sequential solution techniques whereby the BTEs in different directions, frequency bands and polarizations are solved in turn. The BTEs are coupled by the lattice temperature, which is determined from the total energy of all phonon groups. If the Knudsen number is low, inter-BTE coupling becomes too strong for such a sequential procedure to be tenable, and slow convergence rates result. Furthermore, careful resolution of exponentially-thin energy density profiles is necessary at given-temperature boundaries to capture heat fluxes accurately.

A number of publications in the thermal radiation literature have sought to address the convergence issue through the development of solution acceleration schemes. One popular strategy is to advance of the angular-average of the radiative intensity as a way to improve inter-directional coupling. Chui and Raithby [18] proposed a multiplicative correction of the average intensity in the context of the finite volume scheme. However, the scheme was not uniformly convergent. Fiveland and Jessee [19] proposed and evaluated a number of acceleration strategies for the discrete ordinates method. These included the successive over-relaxation method, the mesh rebalance method, and the synthetic acceleration method. The mesh rebalance method, which is similar to [18], was found to perform the best, but its performance deteriorated as the meshbased optical thickness decreased. The method had to be modified to perform rebalance on a coarser mesh than that for the actual solution, so as to keep the rebalance mesh optical thickness greater than unity. Mathur and Murthy [20] proposed a point-coupled multigrid technique to significantly accelerate solution convergence; however, the method is best suited to isotropic scattering problems, and may become too complex for arbitrary scattering kernels. More recently, Hassanzadeh [21] developed the $\mathrm{Q}_{\mathrm{L}}$ algorithm, in which an equation for the average radiative intensity was used to better couple directional intensities. Significant solution acceleration was reported for radiative equilibrium problems. Mathur [22] proposed modifications to the scheme based on a two-level angular multigrid idea which alleviated the loss of overall energy balance in the $\mathrm{Q}_{\mathrm{L}}$ algorithm. Though these new methods hold much promise for phonon transport as well, we are not aware of any use of these acceleration schemes in the BTE literature.

In this paper, we develop a hybrid BTE-Fourier solver which addresses both solution cost and convergence rate. First, we seek to reduce the cost of computation for frequency bands with low band Knudsen number through the use of a modified Fourier conduction equation for these bands. The band-wise Fourier equation contains a scattering term, as well as a firstorder correction at walls to capture temperature jumps. For high Knudsen number (low acoustic thickness) bands, the phonon BTE is solved in the usual sequential fashion, but convergence is relatively straightforward because of the high Knudsen number associated with these bands. The lattice temperature is computed from the combined phonon energies of both BTE and Fourier bands. A finite volume method is 


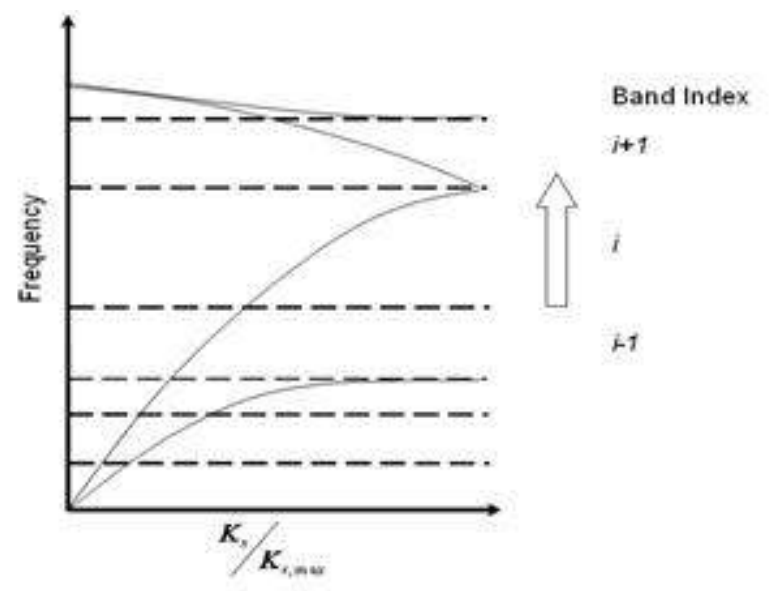

Fig. 1: Dispersion curve in a chosen high-symmetry direction.

employed for both types of equations. The method is applied to a non-gray phonon BTE with both thick and thin bands and shown to perform well.

\section{PHONON BOLTZMANN TRANSPORT EQUATION}

In this section, we present the phonon BTE. We assume an isotropic Brilloiun zone, though this is not integral to the formulation. Typically, dispersion curves in a chosen direction, say [100], are chosen as shown in Fig. 1. The frequency spectrum is discretized into bands, and each band is identified by its frequency $\omega$ and polarization $p$.

\section{Boltzmann Transport Equation}

The steady-state, non-gray BTE for a phonon band of frequency $\omega$ and polarization $p$ under the relaxation time approximation is given by [12]:

$\nabla \cdot\left(\vec{v}_{\omega, p} e_{\omega, p}^{\prime \prime}\right)=\frac{e_{\omega, p}^{0}-e_{\omega, p}^{\prime \prime}}{\tau_{\omega, p}}$

where $\omega$ is the frequency, $p$ is the phonon polarization, $\vec{v}_{\omega, p}$ is the corresponding phonon group velocity and $\tau_{\omega, p}$ the corresponding relaxation $e^{\prime \prime}{ }_{\omega, p}$ is the volumetric energy density per unit solid angle at a given frequency and polarization, and $e_{\omega, p}^{0}$ is the corresponding equilibrium energy density given by a Bose-Einstein distribution [2]. For problems involving relatively small lattice temperature differences, $e_{\omega, p}^{0}$ is given by

$e_{\omega, p}^{0}=\frac{C_{\omega, p}}{4 \pi}\left(T_{L}-T_{r e f}\right)$

Here, $\mathrm{T}_{\mathrm{L}}$ is the lattice temperature and $\mathrm{C}_{\omega, \mathrm{p}}$ is the specific heat associated with the band, and is given by:

$$
C_{\omega, p}=\int_{\Delta K} \hbar \omega \frac{\partial f^{0}}{\partial T} 4 \pi K^{2} d K
$$

Furthermore, the total energy associated with the band is

$$
\int_{4 \pi} e_{\omega, p}^{\prime \prime} d \Omega=C_{\omega, p}\left(T_{\omega, p}-T_{r e f}\right)=4 \pi \bar{e}_{\omega, p}
$$

where $T_{\omega, p}$ is the equilibrium "temperature" associated with the average energy density $\vec{e}_{\omega, p}^{\prime \prime}$ of the band.

\section{Boundary Conditions}

Two thermal boundary conditions are used in this paper for BTE simulations: (i) thermalizing boundaries, and (ii) specularly-reflecting boundaries.

A typical thermalizing boundary at temperature $T_{1}$ is shown in Fig. 2. For phonons going into the domain from the boundary, the energy density for a band of frequency $\omega$ and polarization $p$ is given by:

$e_{1}^{\prime \prime}=\frac{C_{\omega, p}}{4 \pi}\left(T_{1}-T_{r e f}\right)$

At specularly-reflecting boundaries, phonons in a given band in direction $\hat{S}$ incoming from the boundary to the domain are reflected in accordance to [12]:

$$
e_{\omega, p}^{\prime \prime}(\hat{s}, \vec{r})=e_{\omega, p}^{\prime}\left(\hat{s}_{r}, \vec{r}\right)
$$

where $\hat{s}_{r}$ is the specular direction corresponding to $\hat{s}$ and $\vec{r}$ is the position vector on the boundary.

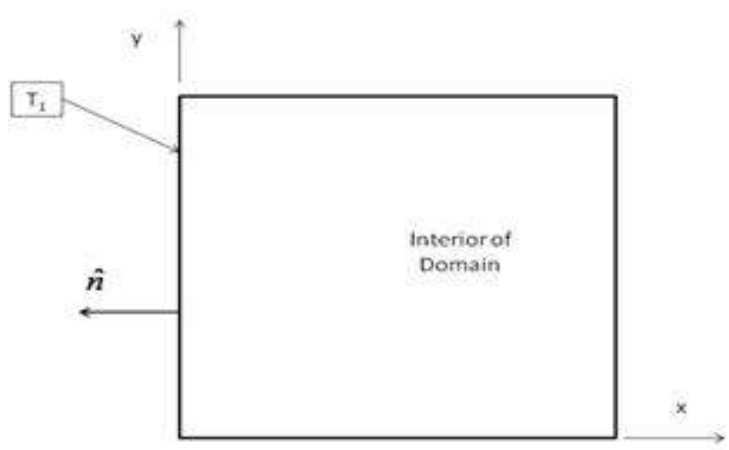

Fig. 2: Thermalizing boundary at temperature $T_{1}$

\section{HYBRID BTE-FOURIER FORMULATION}

The hybrid BTE-Fourier model divides phonon bands into two groups: one group consists of bands described by Eq. (1) and another group in which transport in each band is described by a 
modified Fourier conduction equation. The two groups are coupled through scattering terms and a shared lattice temperature representing the total energy of all bands combined. The choice of which bands are BTE bands and which are Fourier bands is made on the basis of the acoustic thickness $L / v_{\omega, p} \tau_{\omega, p .}$ A cutoff value is chosen, and bands with greater acoustic thickness are modeled as Fourier bands, while those with lower values are solved using the BTE. We describe the hybrid model below.

\section{Fourier Diffusion Equation for Phonons}

Starting with Eqn. (1), we integrate over the solid angle $4 \pi$ to obtain an expression for the heat flux vector $\vec{q}_{\omega, p}$ associated with the band:

$$
\nabla \cdot \vec{q}_{\omega, p}^{\prime \prime}=\frac{C_{\omega, p}}{\tau_{\omega, p}}\left(T_{L}-T_{\omega, p}\right)
$$

For near-equilibrium situations, such as in thermal conduction, we may make the approximation

$$
\nabla \cdot\left(\vec{v}_{\omega, p} e_{\omega, p}^{\prime \prime}\right) \approx \vec{v}_{\omega, p} \cdot \nabla \vec{e}_{\omega, p}^{\prime \prime} \approx C_{\omega, p} \vec{v}_{\omega, p} \cdot \nabla T_{\omega, p}
$$

Using this approximation in Eqn. (1), multiplying it $\vec{v}_{\omega, p}$, and integrating over $4 \pi$ yields the Fourier relationship for the band:

$$
\vec{q}_{\omega, p}^{\prime \prime}=-k_{\omega, p} \nabla T_{\omega, p}
$$

where the band thermal conductivity $k_{\omega, p}$ is given by

$$
k_{\omega, p}=\frac{1}{3} C_{\omega, p} v_{\omega, p}^{2} \tau_{\omega, p}
$$

Combining Eqns. (7), (9) and (10) yields the Fourier equation for the band:

$\nabla \cdot\left(-k_{\omega, p} \nabla T_{\omega, p}\right)=\frac{C_{\omega, p}\left(T_{L}-T_{\omega, p}\right)}{\tau_{\omega, p}}$

\section{Boundary Conditions}

Again, two thermal boundary conditions are considered: (i) thermalizing boundaries, and (ii) specularly reflecting boundaries.

The classical Fourier heat conduction is incapable of predicting temperature jumps at thermalizing boundaries. In order to capture wall temperature jumps, a first-order perturbation is employed. We define $e^{\prime \prime}{ }_{\omega, p}$ as the sum of the average energy of each band and a direction-dependent perturbation:

$$
e^{\prime \prime, p}=\bar{e}_{\omega, p}+\Phi_{\omega, p}(\hat{s})
$$

Here $\Phi_{\omega, p}(\hat{\mathrm{s}})$ is the directionally dependent perturbation and the average band energy is defined in Eqn. (4). We insert Eqn. (12) into the BTE (Eq. 1). Furthermore, we assume that the divergence of $\Phi_{\omega, \mathrm{p}}(\widehat{\mathrm{s}})$ is negligible compared to the divergence of the average band energy density; this can only be true for low Knudsen numbers $(\mathrm{Kn} \$ 0.2)$ or high acoustic thicknesses (> 5 or so). By invoking Eqn. (12), we may obtain an equation for the perturbation at any spatial location for the band as:

$$
\Phi_{\omega, p}=\frac{C_{\omega, p}}{4 \pi}\left[\left(T_{L}-T_{r e f}\right)-v_{\omega, p} \tau_{\omega, p} \nabla \cdot\left(\hat{s} T_{\omega, p}\right)\right]
$$

The above equation expresses the anti-symmetric perturbation to the average energy density implied by the temperature gradient; this assymetry is responsible for creating a heat flux through the domain.

We now examine the thermalizing boundary (Fig. 2). The energy in any band entering the domain from the boundary is assumed to be diffuse and is given by

$e_{1} "=\frac{C_{\omega, p}}{4 \pi}\left(T_{1}-T_{r e f}\right)$

The energy in any band coming to the boundary from the interior depends on $e^{\prime \prime}{ }_{\omega, p}$, as shown in Fig. 2. To find the effective wall temperature $T_{\omega, p, w a l l}$ for the band, the angle averaged energy density at the wall is computed as an average of the incoming and outgoing energies as

$$
\begin{aligned}
e_{1}^{0} & =\frac{1}{4 \pi}\left[\int_{\hat{s} \cdot \hat{n}<0} e_{1}^{\prime \prime} d \Omega+\int_{\hat{s} \cdot \hat{n} \geq 0} e_{\omega, p}^{\prime \prime} d \Omega\right] \\
& =\frac{1}{4 \pi}\left[\frac{e_{1}^{\prime \prime}}{2}+\frac{\vec{e}_{\omega, p}^{\prime \prime}+\Phi_{\omega, p}(\widehat{s})}{2}\right]
\end{aligned}
$$

Here, $\hat{n}$ is the outward-pointing normal at the boundary. Performing the integration and taking advantage Eqns. (10), (12), (13), and (14) while noting that $\hat{s}$ is given by the following:

$\hat{s}=\sin \theta \sin \phi \hat{i}+\sin \theta \cos \phi \hat{j}+\cos \theta \hat{k}$

Where $\theta$ is measured from the positive $\mathrm{z}$ axis and $\phi$ is measured from the positive y axis, we arrive at the following expression: 
$T_{\omega, p, \text { wall }}=\frac{T_{1}-T_{L}}{2}+\frac{v_{\omega, p} \tau_{\omega, p}}{4} \nabla T_{\omega, p} \cdot \hat{n}$

Arranging the terms we can arrive at Robin's condition which is implemented through standard finite volume practice:

$$
\begin{aligned}
& \left(-k_{\omega, p} \nabla T_{\omega, p}\right) \cdot \hat{n}=A T_{\omega, p, \text { wall }}+B \\
& A=-\frac{4}{3} C_{\omega, p} v_{\omega, p} \\
& B=\frac{2 C_{\omega, p} v_{\omega, p}}{3}\left(T_{1}+T_{L}\right)
\end{aligned}
$$

Next we must examine specularly reflecting boundary condition. We will utilize the same procedure which started with Eqn. (15) but this time use Eqn. (6) to define the energy being emitted (really reflected) from the boundary in combination with our perturbation definition (Eqn. 13). Following this, we start with this form:

$$
\begin{aligned}
& e_{\omega, p, \text { wall }}^{0}=\frac{1}{4 \pi}\left[\int_{\hat{s} \cdot \hat{n} \leq 0} \bar{e}^{\prime \prime}+\Phi\left(\hat{s}_{r}\right) d \Omega\right] \\
& +\frac{1}{4 \pi}\left[\int_{\hat{s} \cdot \hat{n}>0} \bar{e}^{\prime \prime}+\Phi(\hat{s}) d \Omega\right]
\end{aligned}
$$

In both of the integrands, the angle average band energy will combine with the first term in the perturbation term to give us the same term we have on the left hand side of Eqn. (19). This leaves us with the sum of the integrations of the perturbation terms, which is noting more than the heat flux. Therefore, we arrive at an adiabatic boundary condition when the walls are specular. It should be noted that if the same approach were taken when examining a diffuse boundary condition, one would still arrive at an adiabitic boundary condition.

\section{Lattice Definition and Temperature Recovery}

For the phonon BTE, energy conservation demands that the right hand side of Eq. (1) summed over all bands and directions must be zero, since scattering is purely redistributive. Thus

$$
\sum_{\omega, p} \frac{4 \pi e_{\omega, p}^{0}-\sum_{4 \pi} e_{\omega, p}^{\prime \prime} \Delta \Omega}{\tau_{\omega, p}}=\sum_{\omega, p} \frac{C_{\omega, p}\left(T_{L}-T_{\omega, p}\right)}{\tau_{\omega, p}}=0
$$

where $\sum_{\omega, p}$ is the summation over all frequency bands and all polarizations. The lattice temperature $T_{L}$ is found from Eqn. (20).
For the hybrid BTE-Fourier model, we employ a similar approach. Now, the scattering terms on the right hand sides of Eqns. (1) and (8) must together sum to zero. Thus:

$$
\sum_{\omega, p}^{B T E} \frac{C_{\omega, p}\left(T_{L}-T_{\omega, p}\right)}{\tau_{\omega, p}}+\sum_{\omega, p}^{\text {Fourier }} \frac{C_{\omega, p}\left(T_{L}-T_{\omega, p}\right)}{\tau_{\omega, p}}=0
$$

The summation in the first term in Eqn. (21) is over all BTE bands, and that in the second term is over all Fourier bands.

We note that Eqns. (1) and (8) are coupled through the lattice temperature $T_{L}$ which appears in the scattering terms in both equations. $T_{L}$ also appears in the specification of thermalizing boundaries for the Fourier bands.

\section{DIMENSIONLESS PARAMETERS}

The governing dimensionless parameters are:

$$
\frac{L}{v_{\omega, p} \tau_{\omega, p}}, \quad \frac{\mathrm{C}_{\omega, \mathrm{p}} / \tau_{\omega, p}}{\sum_{\omega, p} \mathrm{C}_{\omega, \mathrm{p}} / \tau_{\omega, p}}
$$

for each band. The first dimensionless group is the acoustic thickness of the band (inverse of the band Knudsen number). The second dimensionless group, which we call the lattice ratio, results from the lattice temperature equation, Eq. (21).

\section{NUMERICAL METHOD}

\section{Discretization}

A finite volume method is used to solve both the BTE and the Fourier equations $[23,24]$. The present implementation employs structured meshes, though an extension to more general unstructured meshes is straightforward [18]. The same mesh is used for both the BTE and Fourier bands.

The computational domain is divided into rectangular cells or control volumes. For the BTE, we follow discretization procedures similar to those described in $[8,11,17]$. The BTE in any given direction for a band $(\omega, \mathrm{p})$ is integrated over the control volume to yield an energy balance statement for the control volume. For the purposes of this paper, a first-order convective operator is used, though higher-order discretizations are easily admitted. The scattering operator is discretized using a second-order operator. The resulting discrete equation set is solved by traversing the structured mesh in a "streamwise" fashion, dictated by the group velocity vector. For the Fourier bands, the second-order finite volume discretization described in [23] is used. A line-by-line tridiagonal matrix algorithm is used to solve the resulting discrete algebraic set in each band.

\section{Solution Procedure}

Since the Fourier and BTE equations are linear, a direct solution of Eqns. (1), (11) and (21) would produce the final 
solution in one iteration. However, the memory required for the large number of BTE equations is too large to permit direct solutions. Typical solution procedures published for the BTE employ a sequential procedure whereby the BTE in each frequency band and direction is solved sequentially, assuming prevailing values for $\mathrm{T}_{\mathrm{L}}$ in evaluating the energy density $e_{\omega, p}^{0}$. Such a procedure has low memory requirements, and a similar one could be employed here. We would start with a guess of the lattice temperature $T_{L}$ and solve the BTE equations sequentially over the spatial domain, keeping the lattice temperature fixed at its prevailing value. Then the Fourier equations would be solved sequentially, again keeping $\mathrm{T}_{\mathrm{L}}$ at prevailing values. Eq. (21) would then be used to update $T_{L}$. The procedure would be repeated until convergence.

This type of sequential procedure was found to be extremely slow, and was impeded primarily by the explicit update of $T_{L}$. To circumvent this problem, we have developed a partially-implicit procedure. This procedure employs a sequential solution of the BTE equations as before, but augmented by a partially-implicit solution of the Fourier and lattice temperature equations. The computation is initiated with a guess of the lattice temperature. We loop over the BTE bands keeping the lattice temperature fixed at its prevailing value. Once this is complete, the Fourier bands are solved using a block tri-diagonal solver, with a partially-implicit inclusion of the lattice temperature equation (Eq.(21)). The procedure is repeated until a prescribed convergence criterion is met.

The partially-implicit procedure essentially solves the Fourier bands (Eq.(11)) and the lattice temperature (Eq. (21) ) simultaneously, holding the contribution of the BTE bands (i.e., $\mathrm{T}_{\omega, \mathrm{P}}$ in Eq. (21) ) temporarily known at prevailing values. This type of simultaneous solution is feasible for the Fourier bands because the number of Fourier equations is few; it is not feasible for the BTE bands because of the large number of partial differential equations involved. Nevertheless, as will be seen in the next section, it produces significant solution acceleration. This is because $\mathrm{T}_{\mathrm{L}}$ is determined in large part by the thick bands since its value is weighted by the ratio $\mathrm{C}_{\omega, \mathrm{p}} / \tau_{\omega, \mathrm{p}}$, and $\tau_{\omega, \mathrm{p}}$ is small for the acoustically thick Fourier bands. The simultaneous solution of the Fourier bands and $T_{L}$ essentially yields a lattice temperature very close to the final value, and is only mildly retarded by the explicit $\mathrm{T}_{\omega, \mathrm{P}}$ contribution in Eq. (21).

\section{$\underline{\text { Solution Acceleration }}$}

Out of a total of $\mathrm{N}$ bands, if $\mathrm{N}_{\mathrm{F}}$ are the number of Fourier bands, a minimum acceleration of approximately $\mathrm{N} /\left(\mathrm{N}-\mathrm{N}_{\mathrm{F}}\right)$ in $\mathrm{CPU}$ time is expected per iteration, since the Fourier solution

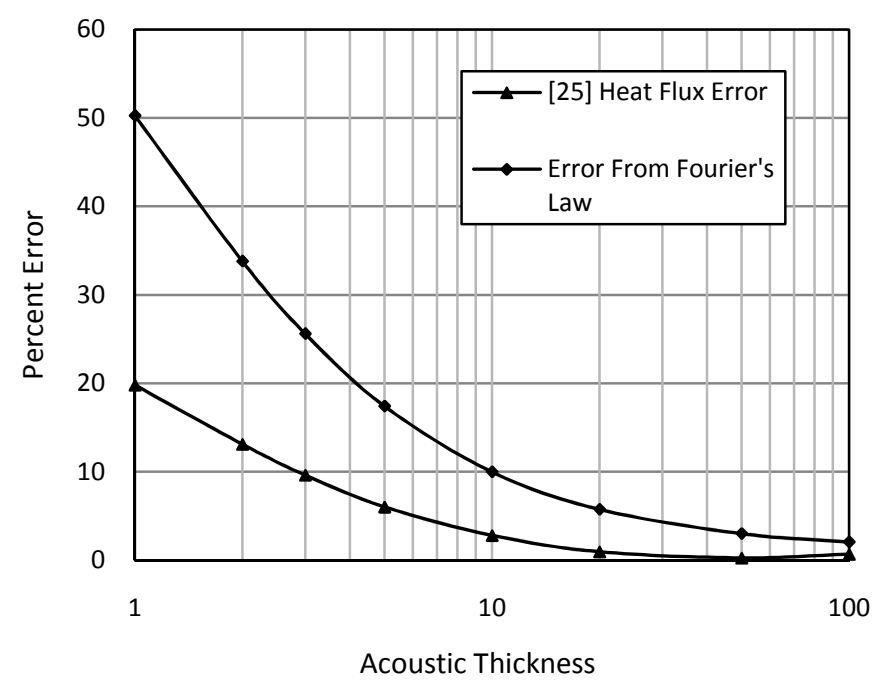

Fig. 4: Percentage error in predicted heat flux using Fourier conduction. Triangles indicate error with respect to [25] when using jump boundary conditions. Diamond symbols indicate the difference between Fourier conduction with jump boundaries and Fourier conduction with Dirichlet boundaries.

cost is negligible compared to the cost of solving the BTE. Any acceleration obtained beyond this value is a result of the algorithmic improvements proposed here.

\section{RESULTS AND DISCUSSION}

In this section, we first establish the correctness of the BTE and Fourier solutions separately, and then consider a two-band problem in which one band is a BTE band and the other is a Fourier band. By varying the acoustic thickness associated with each band, we evaluate the behavior of the hybrid model.

\section{Verification of BTE Solution}

We first solve the classic parallel plate problem of Heaslett and Warming [25]. Here, a one-dimensional slab of width $\mathrm{L}$ is held at temperature $T_{1}$ on its left boundary, and $T_{2}$ on the right boundary. A single BTE band is considered, and has an acoustic thickness of $\mathrm{L} / \mathrm{v}_{\omega, \mathrm{p}} \tau_{\omega, \mathrm{p}}$. Figure 3 shows the dimensionless temperature profile $\left(T-T_{2}\right) /\left(T_{1}-T_{2}\right)$ obtained using the BTE plotted versus dimensionless position. Also on Figure 3 are the exact profiles taken from [25]. The temperature profiles are seen to match the exact solution well. The error in the heat flux for an acoustic thickness of 0.1 is $0.23 \%$, that for a thickness of 1 is $0.20 \%$, while that for thickness of 10 is $5.02 \%$. 


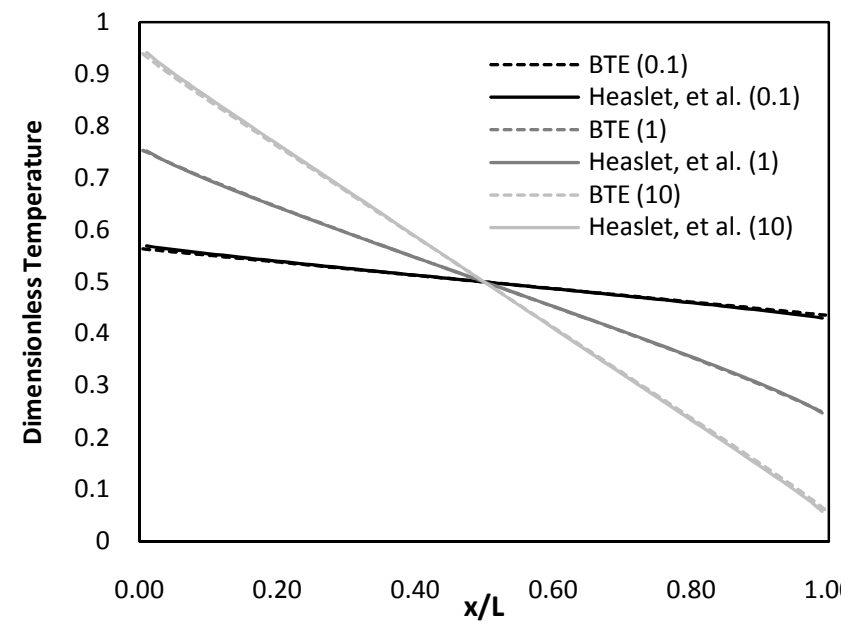

Fig. 3: Comparison of dimensionless temperature profiles of different acoustic thickness (marked in parenthesis) obtained using a single-band BTE with those of Heaslet and Warming [25].

\section{Validation of Fourier Diffusion Equation with Jump Boundary}

\section{Conditions}

Next we seek to establish the correctness of the Fourier diffusion equation with the temperature jump boundary conditions described in previous sections. We consider the onedimensional slab problem again, and only one Fourier band, but vary its acoustic thickness from 1 to 100 . We compare the predicted heat flux with the (exact) solution of Heaslet and Warming [25]. This establishes the acoustic thickness limit beyond which a Fourier approximation with jump boundary conditions may safely be used. We see from Fig. 4 that beyond an acoustic thickness of 5 or so $(K n<0.2)$, the Fourier approximation matches the exact solution with less than $6 \%$ error. Thus, an acoustic thickness of 5 would be a reasonable cut-off value in multi-band simulations. Even for an acoustic thickness as low as 1, an error of only about $20 \%$ is engendered. Thus, jump boundary conditions are a good option for a fast approximation to the BTE.

Also in Figure 5 is the comparison between the heat flux predicted by the Fourier equation with jump boundary conditions and that obtained without jump conditions (i.e., with Fourier conduction with Dirichlet boundary conditions $\mathrm{T}_{1}$ and $\mathrm{T}_{2}$ ). As expected, differences as high as $50 \%$ are engendered at acoustic thickness around unity. At high acoustic thicknesses $(>100)$ the differences between the two types of boundary treatments become small. In the mesoscopic regime, (acoustic thicknesses between 2 and 20) however, the use of jump boundary conditions significantly improves accuracy.

\section{$\underline{\text { Multiband Simulation }}$}

We consider a multiband simulation next, with two bands: one Fourier and one BTE. The Fourier band is always solved using the Fourier conduction equation with jump boundary conditions. The physical domain is again a one-dimensional slab of length $\mathrm{L}$ with boundaries at $\mathrm{T}_{1}$ and $\mathrm{T}_{2}$ respectively. The two bands have acoustic thicknesses $\mathrm{L}_{1} / \mathrm{v}_{1} \tau_{1}$ and $\mathrm{L}_{2} / \mathrm{v}_{2} \tau_{2}$ respectively; these are varied in the problem. Furthermore, the lattice ratio $\frac{C_{1} / \tau_{1}}{C_{1} / \tau_{1}+C_{2} / \tau_{2}}$ is chosen to be 0.90 for the thick band. Computations are performed using the hybrid model, and compared to the corresponding solution using an all-BTE simulation.

Figure 5a shows the heat flux error with respect to the all-BTE solution for the case when the acoustic thickness of the Fourier band is held fixed at 10, while that of the BTE band is varied from 0.1 to 5 . The percentage error is found to be less than $2 \%$ for the range acoustic thicknesses considered. However, we note that there is an increase in error as the BTE band acoustic thickness is increased. This may be explained in the following way. When we have a low acoustic thickness in the BTE band, the overall heat flux is almost entirely dominated by the BTE band, which is the more accurate computation. The errors in the Fourier band computation do not affect the overall outcome to a significant degree. However, as the BTE band acoustic thickness increases, the overall heat flux falls, and the Fourier band contributes proportionally more to the total heat flux. The heat flux error of the Fourier band thus become more apparent and the overall heat flux error increases.

In Figure $5 \mathrm{~b}$ the BTE band is held fixed at an acoustic thickness of 0.5 while the Fourier band acoustic thickness is varied from 1 to 10. Again, the heat flux error with respect to the all-BTE solution is plotted. For low acoustic thicknesses in the Fourier band $\left(\mathrm{L}_{1} / \mathrm{v}_{1} \tau_{1} \sim 1\right)$, the overall error is entirely dominated by the error in the Fourier band solution, which is relatively high (see Fig. 4). However, beyond an acoustic thickness of 5 or so, the heat flux error is seen to fall to values well below 2\%, and decreases as the Fourier band acoustic thickness increases. This is because the assumptions made in deriving the Fourier equation become increasingly true as its acoustic thickness increases. These results indicate that as long as the cutoff acoustic thickness is chosen judiciously, solutions with low error may be obtained with the hybrid model.

\section{Timing Results}


(a)

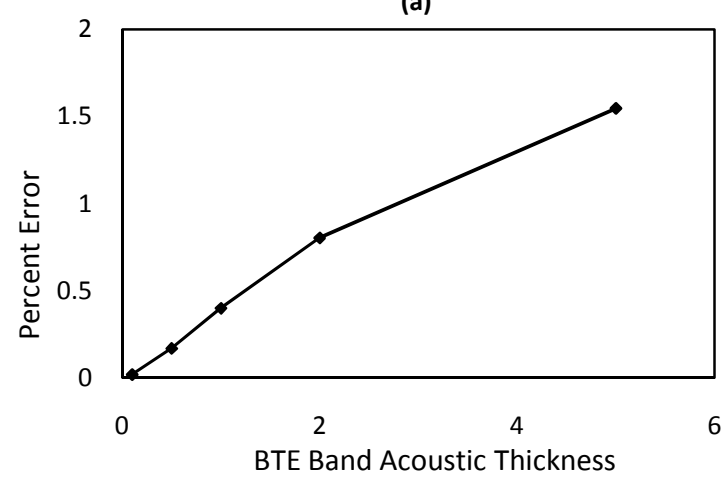

(b)

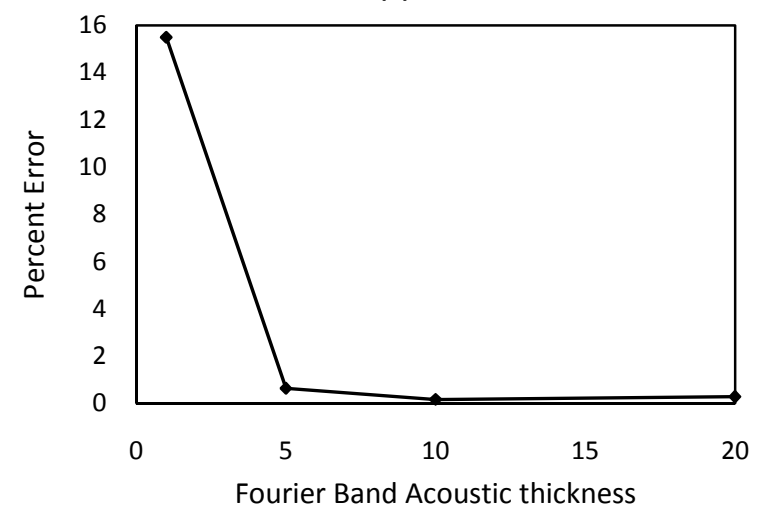

Fig. 5: Heat flux error with respect to all-BTE solution for multi-band simulation. (a) Fourier band fixed acoustic thickness fixed at 10, while varying acoustic thickness of BTE band, and (b) BTE band fixed at acoustic thickness of 5 , while varying acoustic thickness of Fourier band.

We now turn to determination of the amount of solution acceleration afforded by the proposed hybrid solver. The domain used in obtaining timing results is a two-dimensional square slab with Dirichlet boundary conditions on domain boundaries. The left and bottom walls are assumed to be held at a temperature $T_{1}$ while the top and right boundaries are held at a temperature $T_{2}$. Two bands, one solved using the Fourier equation, and the other using the BTE, are again considered. When the hybrid solver is used, the thick band in the discussion below is solved using the Fourier equation, and the thin band is solved using the BTE. The all-BTE solver solves the BTE for both thin and thick bands.

To best demonstrate the strengths and limitations of the hybrid solver we consider several situations. First, we hold the acoustic thickness of the thin band constant at 0.1 while the acoustic thickness of the thick band assumes values of 1,10 , and 100. In the second set of tests considered, the thick band is held fixed at an acoustic thickness of 50, while the thin band has values of $0.1,1$, and 10 . We also wish to examine convergence behavior when we change the lattice ratio, defined in Eq. (22). Three different values of the lattice ratio for the thick band are considered, $\frac{C_{1} / \tau_{1}}{C_{1} / \tau_{1}+C_{2} / \tau_{2}}: 0.90,0.99$, and 0.999. Thus, a total of 18 different cases are considered, each of which is solved by the all-BTE solver and the hybrid solver. The convergence criterion chosen for both solvers requires that the average normalized lattice temperature change between iterations, $\Delta \mathrm{T}_{\mathrm{L}} /\left(\mathrm{T}_{1}-\mathrm{T}_{2}\right)$, fall below $10^{-4}$.

The overall behavior of the hybrid scheme is best explained by first considering the case of a high lattice ratio in the thick band, and a very low one in the thin band, say $\frac{C_{1} / \tau_{1}}{C_{1} / \tau_{1}+C_{2} / \tau_{2}}=0.999$. In this limit, the lattice temperature in Eq. (21) is determined almost entirely by the thick band. Thus, a semi-implicit solution of the Fourier and $T_{L}$ equations is expected to confer a significant advantage and significant solution acceleration over the all-BTE solution results. Furthermore, in this limit, the thin band solution would depend on the $T_{L}$ solution for higher acoustic thicknesses (say 10) but would be entirely decoupled from the $T_{L}$ solution for lower acoustic thicknesses. In either limit, fast convergence is expected, with significant gains over the all-BTE solution. This is shown in the last of the three columns in Fig. 6.

On the other hand, for smaller values of
$\frac{C_{1} / \tau_{1}}{C_{1} / \tau_{1}+C_{2} / \tau_{2}}$, say 0.9 , the thin band contributes to the $\mathrm{T}_{\mathrm{L}}$ determination in Eq. (21), and therefore, the Fourier and BTE bands are coupled. When the thin band acoustic thickness is low, the BTE band converges quickly because it is effectively uninfluenced by $\mathrm{T}_{\mathrm{L}}$ (but not vice versa). Its fast solution helps accelerate the convergence of $T_{L}$, and thus, overall convergence. Therefore, low iteration counts are found in the first column of Fig. 6 for a thin-band acoustic thickness of 0.1 . This effect drops off as the thin band acoustic thickness increases to 10, as seen in Fig. 6.

The same explanations also apply to the cases when the thick band is held at an acoustic thickness of 50, while the thin band is varied from 0.1 to 10 . For the cases when the thinband acoustic thickness is low, the Fourier band is accelerated by the fast convergence of the BTE band, and overall acceleration over the all-BTE solver is very high. The hybrid solver performs to best advantage for high values of $\frac{C_{1} / \tau_{1}}{C_{1} / \tau_{1}+C_{2} / \tau_{2}}$ and high thick-band acoustic thicknesses, for the reasons discussed above.

Figure 7 shows CPU time acceleration obtained using the hybrid solver as compared to the all-BTE solver. The values plotted in Fig. 7 are found by dividing the all-BTE solution time by the hybrid solver time-to-solution. In general, the hybrid solver confers a significant advantage, with acceleration factors ranging from 2-200. The overall trends are explained by the factors discussed above and are not repeated. 


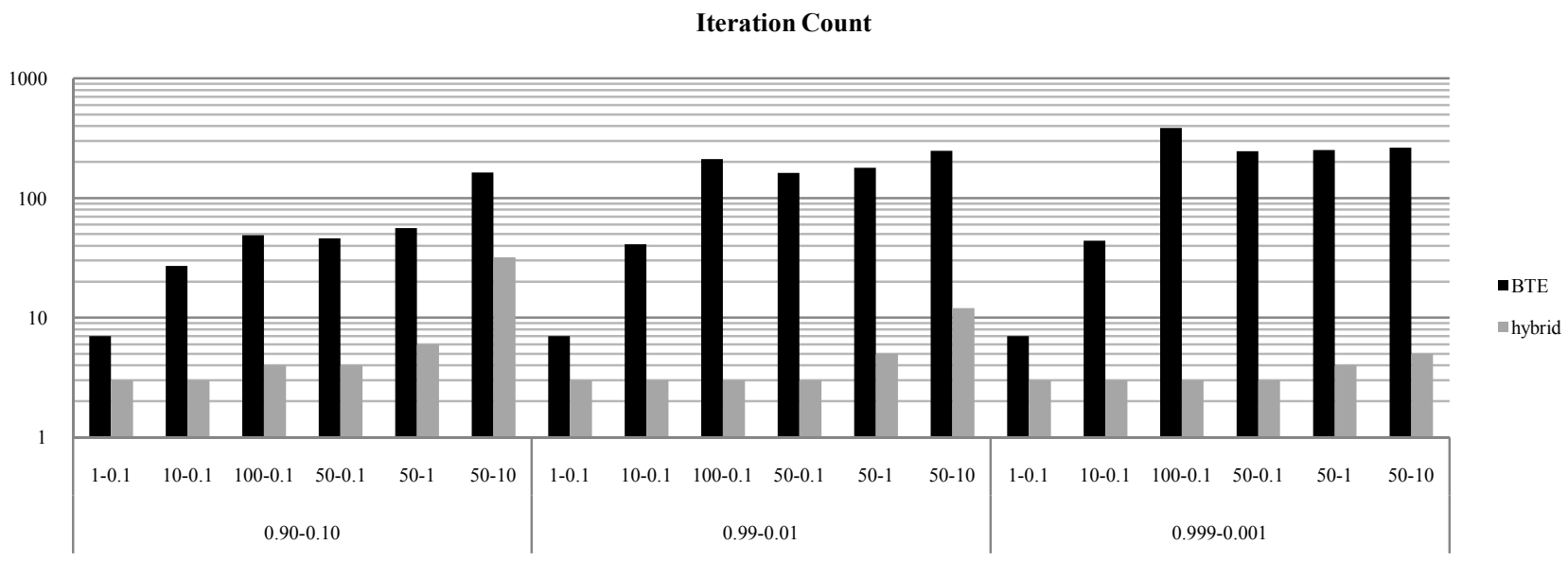

Fig. 6: Comparison of iteration counts for the hybrid and all-BTE solvers for varying thick/thin band acoustic thicknesses, for thickband lattice ratios ranging from 0.90 to 0.999 .

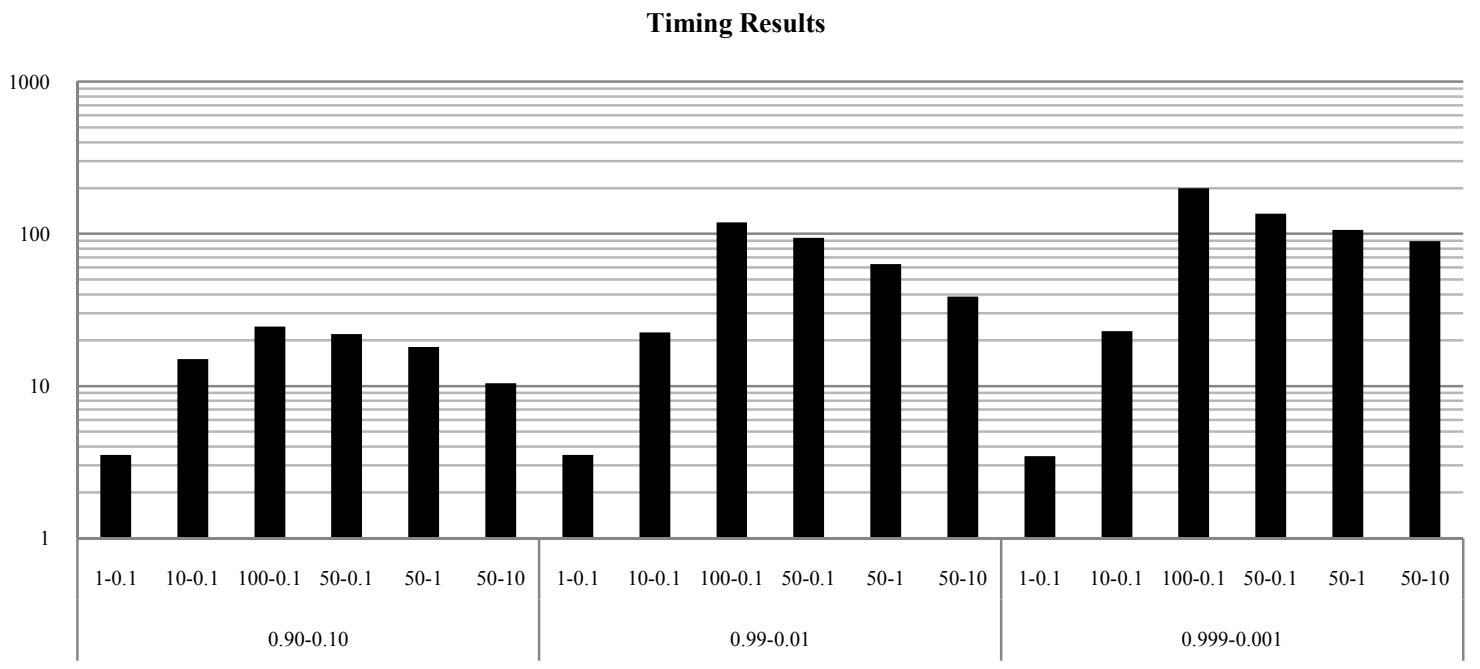

Fig. 7: Acceleration factors achieved by the hybrid solver over the all-BTE solver for different combinations of the thick-thin band acoustic thickness for different thick band lattice ratios.

\section{CONCLUSIONS}

In this paper, we introduced a novel hybrid model for simulating sub-micron thermal transport based on a non-gray phonon BTE formulation. The phonon spectrum is divided into bands, and transport in each band is solved either using a phonon BTE or a Fourier conduction equation with jump boundary conditions. The BTE and the heat diffusion equation are coupled together through a shared lattice temperature which is computed by enforcing overall energy balance. A novel partially-implicit solution procedure is devised which seeks to better couple the Fourier and lattice temperature calculation. This procedure, coupled with the fact that the Fourier conduction equation is significantly less expensive to solve than the BTE, greatly reduces computation time. Our tests indicate that there is little cost to accuracy if the cutoff acoustic thickness is chosen with care. Studies are underway to apply the new method to sub-micron thermal transport problems in microelectronics and other applications. 


\section{ACKNOWLEDGMENTS}

This material is based upon work supported in part by the Department of Energy (National Nuclear Security Administration), under Award Number DE-FC52-08NA28617. Partial support from the DARPA-funded IMPACT Center is also acknowledged.

\section{REFERENCES}

[1] International Technology Roadmap for Semiconductors (ITRS), 2002, 2002 Update. [Online]. Available: http://www.itrs.net/Links/2002Update/2002Update.pdf.

[2] C. Kittel. 1996. Introduction to Solid State Physics. John Wiley, New York.

[3] McGaughey A.J.H. and M. Kaviany, 2004. "Phonon Transport in Molecular Dynamics Simulations: Formulation of Thermal Conductivity Prediction." Physical Review B, 69. pp. $1-12$.

[4] Sun L., J.Y. Murthy, 2006. "Domain Size Effects in Molecular Dynamics Simulation of Phonon Transport in Silicon.” Applied Physics Letters 89 (17) 171919.

[5] Henry A.S and G. Chen, 2008. "Spectral Phonon Transport Properties of Silicon Based on Molecular Dynamics Simulations and Lattice Dynamics." Journal of Computational and Theoretical Nanoscience, 5, pp. 1-12.

[6] Zhang W., T. S. Fisher, Mingo N., 2007. "The Atomistic Green's Function Method: an Efficient Simulation Approach For Nanoscale Phonon Transport." Numerical Heat Transfer Part B, 51, pp. 333-349

[7] Datta S., 2005, Quantum Transport: Atom to Transistor, Cambridge University Press, Cambridge, UK.

[8] Narumanchi S.V.J., J.Y. Murthy, J.Y., and C.H. Amon, 2004. "Submicron Heat Transport Model in Silicon Accounting for Phonon Dispersion and Polarization." ASME Journal of Heat Transfer, 126, pp. 946-955.

[9] Singh, D., Murthy, J. Y., Fisher, T. S., 2008. "Thermal Transport in Finite Sized Nanocomposites." SHTC 200856385. Proceedings of SHTC. Jacksonville, Florida: ASME.

[10] Murthy, J. Y., Narumanchi S. V. J., Pascual-Gutierrez J. A. Wang T., Ni C. Mathur S. R., 2005. "Review of Multiscale Simulation in Sub-micron Heat Transfer." International Journal for Multiscale Computational Engineering.

[11] Ni, C., 2009. "Phonon Transport Models for Heat Conduction in Sub-Micron Geometries with Applications to Microelectronics.” Purdue University, West Lafayette.
[12] Murthy, J., Mathur, S., 2003. "An Improved Computational Procedure for Sub-micron Conduction." Journal of Heat Transfer. 125 (5) pp. 904-911

[13] Sverdrup P.G., 2000, "Simulation and Thermometry of Sub-continuum Heat Transport in Semiconductor Devices." Stanford University, Stanford.

[14] Ju Y.S., 1999. "Microscale Heat Conduction in Integrated Circuits and Their Constituent Films." Stanford University, Stanford.

[15] Jacoboni, C., and Lugli, P., 1989. The Monte Carlo method for semiconductor device simulation. Springer Verlag, Vienna.

[16] Modest, M. Radiative Heat Transfer. Academic Press, 2003.

[17] Wang T.J., 2007. "Sub-micron Thermal Transport in Ultrascaled Metal Oxide Semiconductor (MOS) Devices." Purdue University, West Lafayette

[18] E.H. Chui and G.D. Raithby, 1992. "Implicit Solution Scheme to Improve Convergence Rate in Radiative Transfer Problems," Numerical Heat Transfer, 22, pp. 251-272.

[19] W.A. Fiveland and J.P. Jessee, 1996. "Acceleration Schemes for the Discrete Ordinates Method," Journal of Thermophysics and Heat Transfer 10 (3) pp. 445-451.

[20] S.R. Mathur, and J.Y. Murthy, 1999. "Coupled Ordinate Method for Multi-Grid Acceleration of Radiation Calculations," Journal of Thermophysics and Heat Transfer, 13 (4) pp. 467-473.

[21] P. Hassanzadeh 2007. "Efficient Computational Thermal Radiation." University of Waterloo

[22] S.R. Mathur, 2008. "Efficient Radiation Simulation in Complex Geometries with Applications to Planetary Entry." NASA Final Report, Contract \#NNX08CC61P.

[23] Patankar, S. V., 1980. Numerical Heat Transfer and Fluid Flow. Taylor \& Francis

[24] Chai J.C., H.S. Lee, and S.V. Patankar, 1994, "Finite volume method for radiation heat transfer", Journal of Thermophysics and Heat Transfer 8 (3) pp. 419-425.

[25] M. A. Heaslet and R. F. Warming, 1964. "Radiative Transport and Wall Temperature Slip in an Absorbing Planar Medium." International Journal of Heat and Mass Transfer 8 pp. 979-994. 